Document downloaded from:

http://hdl.handle.net/10251/166019

This paper must be cited as:

Vallejo-Castro, L.; Nguyen, D.; Bohata, J.; Ortega Tamarit, B.; Zvanovec, S. (2020). M-QAM signal transmission at the photonically generated K-band over thermal-induced turbulent FSO links with different turbulence distributions. Applied Optics. 59(16):4997-5005. https://doi.org/10.1364/AO.390103

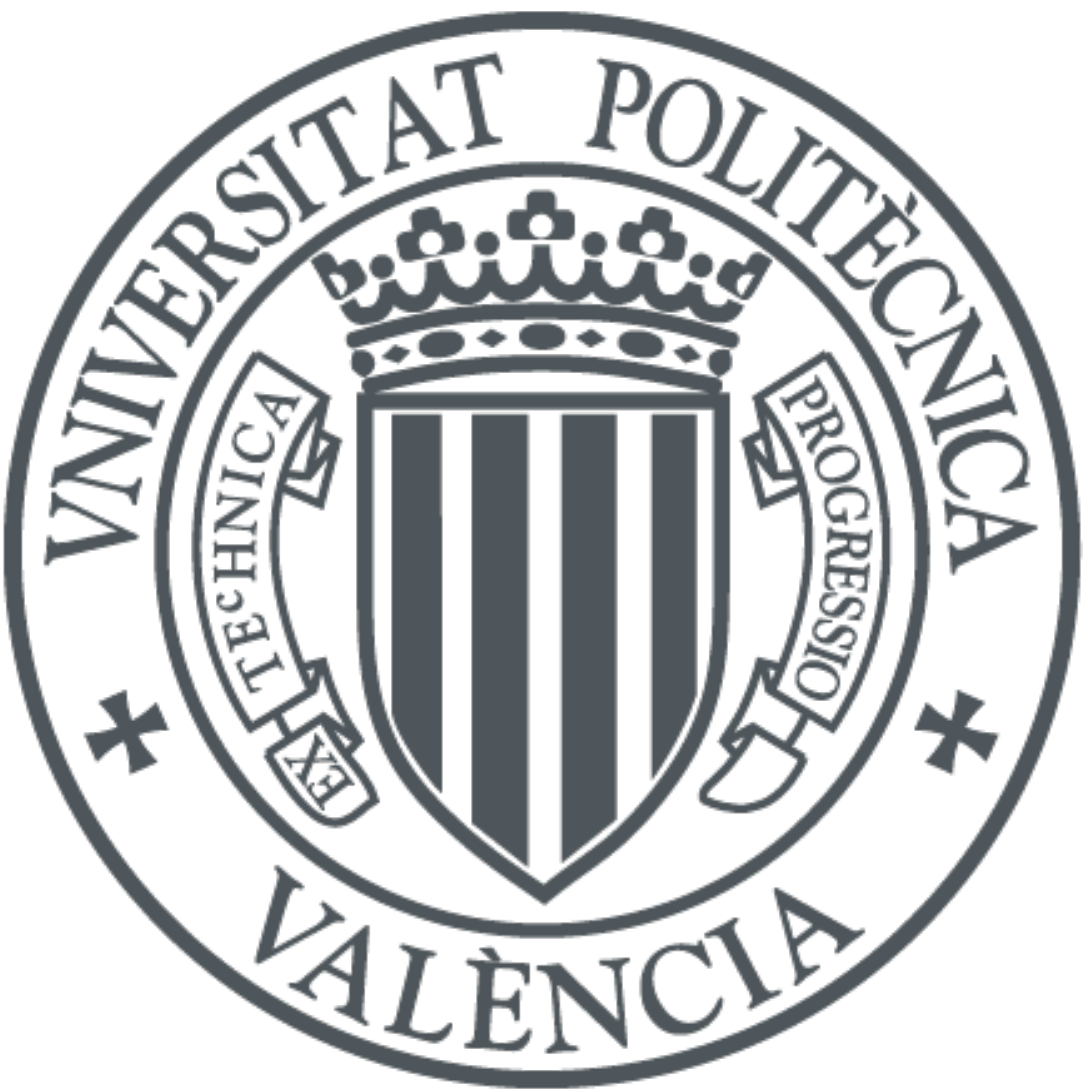

The final publication is available at

https://doi.org/10.1364/AO.390103

Copyright The Optical Society

Additional Information 


\title{
M-QAM signals transmission at the photonically generated K-band over thermal-induced turbulent FSO links with different turbulence distributions
}

\author{
Luis Vallejo1, Dong-Nhat Nguyen², Jan Bohata ${ }^{2}$, Beatriz \\ OrTEGA ${ }^{1}$ And STANISLaV ZVANOVEC ${ }^{2}$ \\ ${ }^{1}$ Instituto de Telecomunicaciones y Aplicaciones Multimedia, Universitat Politecnica de Valencia, \\ Camino de vera, s/n 46022 Valencia (Spain) \\ ${ }^{2}$ Department of Electromagnetic Field, Faculty of Electrical Engineering, Czech Technical \\ University in Prague, Technicka 2, 16627 Prague (Czech Republic) \\ *bortega@dcom.upv.es
}

\begin{abstract}
:
We present a theoretical and experimental study on the impact of different thermal-induced free space turbulence distributions on the M-quadrature amplitude modulation (QAM) signal transmission in radio frequency K-band over hybrid optical links of standard single mode fiber (SSMF) and free-space optics (FSO). Frequency multiplication using an external intensity modulator biased at the null transmission point has been employed to photonically generate the radio signals at the frequency of $25 \mathrm{GHz}$ falling to considered frequency band for fifth-generation (5G) mobile networks. Moreover, extensive simulations have been performed for $10 \mathrm{~Gb} / \mathrm{s}$ with 4-, 16- and 64-QAM over $5 \mathrm{~km}$ of SSMF and $500 \mathrm{~m}$ long FSO channel under scenarios with different turbulence levels and distributions whereas proof-of-concept experiments have been conducted for $20 \mathrm{MHz}$ with 4- and 64-QAM over $5 \mathrm{~km}$ of SSMF and $2 \mathrm{~m}$ long FSO channel under turbulence conditions. Both theoretical and experimental systems have been analysed in terms of EVM performance showing feasible transmission over the hybrid links in the received optical power range. Non-uniform turbulence distributions are shown to have different impact on M-QAM modulation formats, i.e. turbulence distributions with higher strength in the middle of the FSO link evince $1.9 \mathrm{~dB}$ penalty when using 64-QAM signals compared to $1.3 \mathrm{~dB}$ penalty using 4-QAM signals, whereas higher penalties have been measured when 4-QAM format is transmitted over turbulence distributions with larger magnitude in the second half of the FSO link. The results have been validated by theoretical predictions and lead to practical consequences on future networks' deployment.
\end{abstract}

Index Terms: Free space optical communications, turbulence, millimeter-waves, optical fibers

\section{Introduction}

The enormous growth of mobile data traffic prompts the evolution of legacy technologies, i.e. fourth-generation mobile communications, $4 \mathrm{G}$, towards the fifth-generation, $5 \mathrm{G}$, which supports the new challenges, such as high data rate, high spectral efficiency or low latency [1]. The spectrum below $6 \mathrm{GHz}$ (FR1), which is currently congested, carries among others the traditional cellular communications traffic whereas higher frequency bands in range FR2 (24.25 - $52.60 \mathrm{GHz})$ [2] are aimed at providing short range and very high throughput capability for $5 \mathrm{G}$ networks. Millimeter-waves $(\mathrm{mmW})$ in the given frequency range will significantly cover these challenges.

Radio-over-fiber, RoF, which is well known as a hybrid technology combining optical and radio signals, plays a key role in future networks deployment due to its main advantages, such as low attenuation, immunity to radio frequency interference, 
transparency to modulation formats, high capacity, flexibility and dynamic resource allocation [3]. In the legacy network architecture, the baseband unit (BBU) and the antenna are located close to each other with the reduced data rate. However, cloud radio access network (C-RAN) architecture includes a fronthaul link between the central station (CS) remote base stations (BS), providing a higher data rate and less power consumption. RoF in 5G C-RAN [4] is an excellent solution for such fronthaul link, which can transport mmW signals carrying data rates of $\mathrm{Gb} / \mathrm{s}$ along tens of kilometres of optical fiber (OF) [5].

Furthermore, microwave photonics allows to generate and deliver mmW signals with low phase noise and frequency tunability [6, 7]. Among many schemes proposed in the literature, the external modulation with optical frequency multiplication between modulated sidebands leads to a very convenient approach in terms of cost and complexity. Moreover, frequency doubling, quadrupling, up to 8-tupling [8] have been demonstrated, allowing to further reduce the electric bandwidth requirements.

Free space optics (FSO) systems, which use the air as a transmission medium, are very attractive compared to traditional wireless radio frequency (RF) systems. FSO uses extremely narrow laser beams allowing high reuse factor, inherent security and robustness to electromagnetic interference. Besides, FSO employs THz carrier frequencies which do not require license fees [9] and is an excellent alternative in areas where the OF cannot be installed. Note that FSO losses are much lower than RF radiation and propagation losses due to high directivity. For example, the atmospheric attenuation for signal wavelengths in the transmission window $1520-1600 \mathrm{~nm}$ is less than $0.2 \mathrm{~dB} / \mathrm{km}$ with clear visibility [10], whereas the RF losses, strongly dependent on the electrical frequency, lead to up to e.g. $60 \mathrm{~dB}$ over $1 \mathrm{~m}$ at $25 \mathrm{GHz}$ frequency [11] with particular antenna. However, several atmospheric effects, such as fog or turbulence, must be also considered in the case of FSO transmission [12]. Misalignments, so-called pointing errors, either due to buildings thermal expansion, wind or weak seismic activity, amongst others, must also be considered and further compensated $[13,14]$.

In particular, FSO is affected by atmospheric turbulence created by temperature gradients [15-17] occurring naturally in an outdoor environment. However, the thermal distribution can vary significantly along the FSO link especially in the cities as a result of the presence of the sources of hot and cold air, e.g. rivers or highways beneath optical path. More specifically, the performance of FSO links, which are due the flexibility widely deployed in dense urban areas, is affected by the buildings heating, air conditioning, presence of watercourse or wind circulation in street corridors, etc. So far, only uniform turbulence regimes have been thoroughly studied in the literature [18], whereas previous experimental measurements recently published in [19] have confirmed the need for studying the impact of different atmospheric turbulence distributions on the performance of radio-over-FSO (RoFSO) links.

In this paper, we present a theoretical and experimental study of the impact of nonuniform turbulent distribution along the FSO link on the transmission of M-quadrature amplitude modulation (QAM) signals with $\mathrm{M}=4,16$ and 64 in $\mathrm{K}$-band (25 GHz), which are photonically generated by using an optical external intensity modulator biased at null transmission point. The paper is structured as follows: section 2 includes the fundamentals of the approach, simulation results are provided in section 3 and section 4 presents the proof-of-concept with experimental results. Finally, the conclusion section outlines the main results obtained throughout the paper.

\section{Fundamentals}

The proposed system layout of M-QAM signal transmission at $25 \mathrm{GHz}$ over a hybrid standard single mode fiber (SSMF) and FSO link for simulation is shown in Fig. 1. 


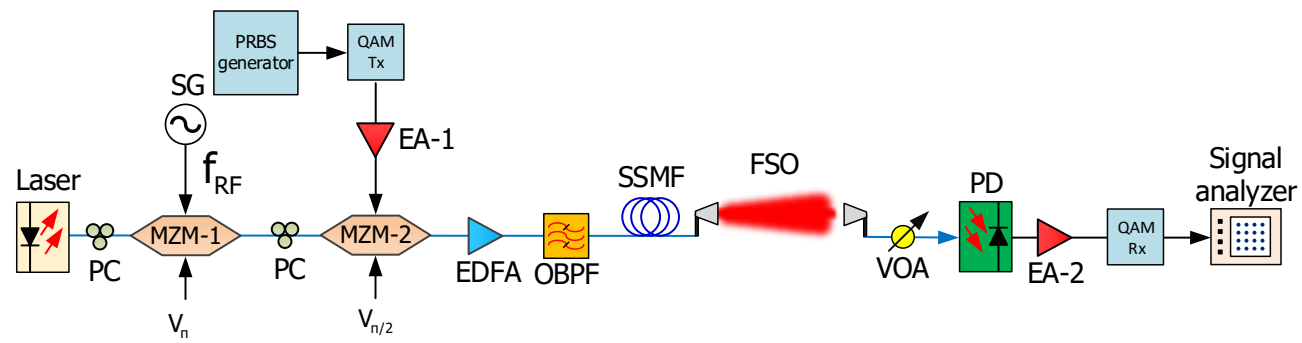

Fig. 1. System layout. PC: polarization controller, MZM: Mach-Zehnder modulator, SG: signal generator, PRBS: pseudorandom binary sequence, QAM: quadrature amplitude modulation, Tx: transmitter, EA: electrical amplifier, EDFA: erbium doped fiber amplifier, OBPF: optical bandpass filter, SSMF: standard single mode fiber, FSO: free space optics, VOA: variable optical attenuator, PD: photodetector, Rx: receiver.

In this architecture, an optical carrier emitted by the laser, whose state of polarization is adjusted by a polarization controller (PC), can be expressed as:

$$
E(t)=E_{0} \cos \left[\omega_{0} t+\phi_{0}(t)\right]
$$

where $E(t)$ is the electric field, $E_{0}, \omega_{0}$ and $\phi_{o}(t)$ are the electric field amplitude, the angular frequency and the phase of the optical carrier with random fluctuation, respectively.

The signal generator ( $\mathrm{SG}$ ) generates an electrical clock signal given as:

$$
V(t)=V_{e} \cos \left[\omega_{e} t+\phi_{e}(t)\right]
$$

where $V(t)$ is the voltage, $V_{e}$ and $\omega_{e}$ are the voltage amplitude and angular frequency, respectively. $\phi_{e}(t)$ represents an independent random process that introduces phase fluctuation of the electrical signal.

The modulated optical signal at the Mach-Zehnder modulator (MZM-1) output is given by [20]:

$$
E_{\text {out }-M Z M 1}(t)=\alpha_{M Z M} E_{0} \cos \left\{\frac{\phi_{D C}}{2}+\beta_{1} \cos \left[\omega_{e} t+\phi_{e}(t)\right]\right\} \cos \left[\omega_{0} t+\phi_{0}(t)\right],
$$

where $\alpha_{M Z M}$ is the insertion loss of the MZM, $\phi_{D C}$ is the constant phase shift determined by the dc bias voltage and $\beta_{1}$ is the modulation index, which can be expressed as:

$$
\beta_{1}=\frac{\pi}{V_{\pi_{1}}} \cdot \frac{V_{e}}{2},
$$

where $V_{\pi_{1}}$ is the half-wave voltage of the MZM-1. When $\phi_{D C}=(2 k-1) \pi$, all even-order sidebands are suppressed and the second and higher-order optical sidebands can be ignored provided an appropriate electrical drive signal is selected. Then, the output of MZM-1 can be expressed as:

$$
\begin{aligned}
E_{\text {out }-M Z M 1}(t)= & -\alpha_{M Z M} E_{0} J_{1}\left(\beta_{i}\right)\left\{\cos \left[\left(\omega_{0}-\omega_{e}\right) t+\phi_{0}(t)-\phi_{e}(t)\right]+\right. \\
& \left.\cos \left[\left(\omega_{0}+\omega_{e}\right) t+\phi_{0}(t)+\phi_{e}(t)\right]\right\}
\end{aligned}
$$

where $J_{1}(\cdot)$ is the first-order Bessel function of the first kind.

The optical signal is then launched into the MZM-2 and modulated by the data information, $A(t)$, i.e. an amplified pseudo-random binary sequence (PRBS) M-QAM signal at the linear bias point. The output in this case is given as:

$$
E_{\text {out }-M Z M 2}(t)=A(t) E_{\text {out }-M Z M 1}(t) \text {. }
$$


An erbium-doped fiber amplifier (EDFA) is then used to compensate losses by the gain and its output signal is filtered out by an optical bandpass filter (OBPF) to reduce the amplified spontaneous emission (ASE) over a narrow optical bandwidth. The signal is subsequently launched into the SSMF, where the optical carrier suppressed mmW signal suffers only the time shifting of the codes and is considerably more immune to this fading effect [21]. By considering the Taylor expansion of the propagation constant, $\beta(\omega)$ of the fundamental mode in the SSMF:

$$
\beta\left(\omega_{0} \pm \omega_{e}\right)=\beta\left(\omega_{0}\right) \pm \omega_{e} \beta^{\prime}\left(\omega_{0}\right)+\frac{1}{2} \omega_{e}^{2} \beta^{\prime \prime}\left(\omega_{0}\right)+\cdots,
$$

the code distortion can be approximated as [21]:

$$
A_{ \pm 1}=\alpha_{M Z M} J_{1}\left(\beta_{1}\right) A\left(t-\frac{\beta\left(\omega_{0} \pm \omega_{e}\right) z}{\left(\omega_{0} \pm \omega_{e}\right)}\right) e^{-\gamma z},
$$

where $\gamma$ is the attenuation constant and $z$ is the length of the SSMF.

After transmission over optical fiber link, the M-QAM signal is then propagated over the FSO channel (i.e. last mile access). The FSO part is based on three subsystems: a transmitter telescope, free space and a receiver telescope. The FSO loss, $\alpha_{F S O}$ includes the attenuation due to the atmospheric propagation effects and geometrical loss due to the spreading of the transmitted beam between the transmitter and the receiver, and can be determined according to [10]:

$$
\alpha_{F S O}=\frac{P_{R x}}{P_{T x}}=\frac{d_{R}^{2}}{\left(d_{T}+\theta \mathrm{R}\right)^{2}} 10^{-\alpha_{a t m} \frac{R}{10}},
$$

where $d_{R}$ and $d_{T}$ are diameters of the receiver and transmitter aperture in meter, $\theta$ is the beam divergence in milli-radians, $R$ is the FSO range in kilometers and $\alpha_{\text {atm }}$ is the atmospheric attenuation in $\mathrm{dB} / \mathrm{km}$. Moreover, FSO is widely affected by turbulence leading to significant fluctuations in the transmitted optical power which contribute to the signal degradation. Following the Gamma-Gamma atmospheric turbulence model [22], the scintillations of the signal in the channel having the length $L$ can be characterized by the log-amplitude variance, commonly named as Rytov variance $\sigma_{R}^{2}$, which can be calculated for a uniform refractive index structure parameter $\left(C_{n}^{2}\right)$ as:

$$
\sigma_{R}^{2}=1.23 k^{7 / 6} C_{n}^{2} L^{11 / 6} \text {. }
$$

The $C_{n}^{2}$ parameter, which determines the strength of the turbulence, is given by:

$$
C_{n}^{2}=\left(79 \cdot 10^{-6} \cdot \frac{P_{a}}{T^{2}}\right)^{2} C_{T}^{2},
$$

where $P_{a}$ is the atmospheric pressure in millibars, $T$ is the absolute temperature in Kelvin and $C_{T}^{2}$ is the temperature structure parameter, which is defined as:

$$
C_{T}^{2}=\frac{\left(T_{1}-T_{2}\right)^{2}}{L_{P}^{2 / 3}}
$$

$T_{1}$ and $T_{2}$ are the temperatures at two separated points by a distance $L_{p}$.

Therefore, the probability of a given optical power after transmission over a FSO link, $P_{F S O}$, is given by the following distribution:

$$
\operatorname{Prob}\left(P_{F S O}\right)=\frac{2(\alpha \beta)^{(\alpha+\beta) / 2-1}}{\Gamma(\alpha) \Gamma(\beta)} K_{\alpha-\beta}\left(2 \sqrt{\alpha \beta} P_{F S O}\right),
$$

where $\Gamma(\cdot)$ Is the Gamma function, $K_{\alpha-\beta}(\cdot)$ is the second kind modified Bessel function and $1 / \alpha$ and $1 / \beta$ are the variances of small and large scale eddies, which are calculated from the Rytov variance as: 


$$
\begin{aligned}
& \alpha=\exp \left[\frac{0.49 \sigma_{R}^{2}}{\left(1+1.11 \sigma_{R}^{12 / 5}\right)^{5 / 6}}\right]-1 \\
& \beta=\exp \left[\frac{0.51 \sigma_{R}^{2}}{\left(1+0.69 \sigma_{R}^{12 / 5}\right)^{5 / 6}}\right]-1
\end{aligned}
$$

Finally, the signal is launched into a variable optical attenuator (VOA), which allows to vary the optical power just before detection at the photodetector (PD). After optoelectronic conversion, the M-QAM signal data is carried by the microwave signal at $2 \omega_{e}$ angular frequency, as a result of the beating between the two first-order sidebands obtained in eq. (5). The resulting photocurrent can be calculated similarly as introduced in [21] as:

$$
I_{2 \omega_{e}}(z, t)=2 \Re \alpha_{F S O} A_{+1} A_{-1} \cos \left[2 \omega_{e} t-2 \omega_{e} \beta^{\prime}\left(\omega_{o}\right) z\right],
$$

where $\Re$ is the responsivity of the photodetector. The photocurrent is further electrically amplified and properly analysed to evaluate the system performance. The main simulation parameters of the proposed hybrid system are given in Table 1 .

\section{Simulation results}

In this section, we evaluate the performance of the system depicted in Fig. 1 with the parameters detailed in Table 1 under different turbulence scenarios by using a cosimulation of Optisystem and Matlab software. Uniform turbulence have been defined according to weak $\left(\sigma_{R}^{2}<<1\right)$, moderate $\left(\sigma_{R}^{2} \approx 1\right)$ and strong $\left(\sigma_{R}^{2}>>1\right)$ regimes (denoted as $\mathrm{U}$ scenarios: U1, U2, U3), whereas non-uniform scenarios include up to three different sections (denoted as N-scenarios: N1, N2, N3) with varying turbulence level along the link, as detailed in Table 2. It is worth to mention that all the $\mathrm{N}$-scenarios were set to have a similar average turbulence value as U2 uniform scenario for the sake of comparison.

Table 1. Simulation parameters.

\begin{tabular}{|l|l|c|}
\hline \multicolumn{2}{|l|}{ Parameter } & Value \\
\hline \multirow{2}{*}{ Laser } & Wavelength & $1550 \mathrm{~nm}$ \\
\cline { 2 - 3 } & Output power & $14 \mathrm{dBm}$ \\
\hline MZM-1 & Switching RF voltage & $4 \mathrm{~V}$ \\
\hline SG & Carrier frequency & $12.5 \mathrm{GHz}$ \\
\hline \multirow{2}{*}{ PRBS } & Bit rate & $10 \mathrm{~Gb} / \mathrm{s}$ \\
\cline { 2 - 3 } & Order & 16 \\
\hline MZM-2 & Switching RF voltage & $4 \mathrm{~V}$ \\
\hline \multirow{2}{*}{ Modulation formats } & \multicolumn{2}{|c|}{$4-, 16-, 64-\mathrm{QAM}$} \\
\hline \multirow{2}{*}{ OBPF } & Output power & $13 \mathrm{dBm}$ \\
\cline { 2 - 3 } & Noise figure & $3 \mathrm{~dB}$ \\
\hline \multirow{3}{*}{ SSMF } & Bandwidth & $1.5 \mathrm{~nm}$ \\
\cline { 2 - 3 } & Optical insertion loss & $1 \mathrm{~dB}$ \\
\hline \multirow{5}{*}{ FSO } & Length & $5 \mathrm{~km}$ \\
\cline { 2 - 3 } & Dispersion & $17 \mathrm{ps} \cdot \mathrm{nm} / \mathrm{km}$ \\
\cline { 2 - 3 } & Attenuation & $0.2 \mathrm{~dB} / \mathrm{km}$ \\
\hline & Tx aperture & $2.6 \mathrm{~cm}$ \\
\cline { 2 - 3 } & Tx loss & $1 \mathrm{~dB}$ \\
\cline { 2 - 3 } & Rx aperture & $5 \mathrm{~cm}$ \\
\cline { 2 - 3 } & Rx loss & $1 \mathrm{~dB}$ \\
\hline
\end{tabular}




\begin{tabular}{|l|l|c|}
\hline \multirow{2}{*}{ VOA } & Beam divergence & $0.25 \mathrm{mrad}$ \\
\cline { 2 - 3 } & Length & $500 \mathrm{~m}$ \\
\hline \multirow{2}{*}{$P D$} & Insertion loss & $0-10 \mathrm{~dB}$ \\
\hline EA-1 & Responsivity & $0.45 \mathrm{~A} / \mathrm{W}$ \\
\cline { 2 - 3 } & Dark current & $5 \mathrm{nA}$ \\
\hline$E A-2$ & Gain & $9.1 \mathrm{~dB}$ \\
\hline
\end{tabular}

Fig. 2 shows the spatial distribution of turbulence along the FSO link, according to the parameters specified in Table 2 for different scenarios.
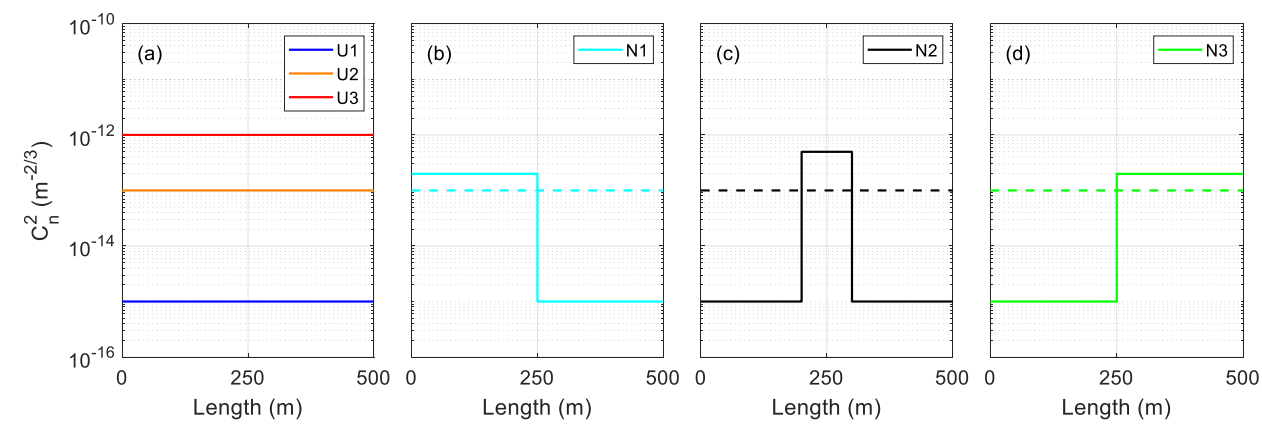

Fig. 2. $C_{n}^{2}$ distribution for different scenarios: (a) U1, U2 and U3, (b) N1, (c) N2 and (d)

N3. Dashed line represents the mean value in each scenario.

Table 2. Definition of scenarios

\begin{tabular}{|c|c|c|c|c|c|}
\hline \multicolumn{2}{|l|}{ Scenario } & Distance & $\mathrm{C}_{\mathrm{n}}^{2}\left(\mathrm{~m}^{-2 / 3}\right)$ & Averaged $C_{n}^{2}\left(m^{-2 / 3}\right)$ & Rytov variance \\
\hline \multirow{3}{*}{ Uniform } & U1 & $500 \mathrm{~m}$ & $10^{-15}$ & $10^{-15}$ & 0.0056 \\
\hline & $\mathrm{U} 2$ & $500 \mathrm{~m}$ & $10^{-13}$ & $10^{-13}$ & 0.5587 \\
\hline & U3 & $500 \mathrm{~m}$ & $10^{-12}$ & $10^{-12}$ & 5.5869 \\
\hline \multirow[t]{7}{*}{ Non-uniform } & N1 & $250 \mathrm{~m}$ & $2 \cdot 10^{-13}$ & \multirow{2}{*}{$10^{-13}$} & \multirow{2}{*}{0.5587} \\
\hline & & $250 \mathrm{~m}$ & $10^{-15}$ & & \\
\hline & \multirow[t]{3}{*}{$\mathrm{N} 2$} & $200 \mathrm{~m}$ & $10^{-15}$ & \multirow{3}{*}{$10^{-13}$} & \multirow{3}{*}{0.5587} \\
\hline & & $100 \mathrm{~m}$ & $5 \cdot 10^{-13}$ & & \\
\hline & & $200 \mathrm{~m}$ & $10^{-15}$ & & \\
\hline & \multirow[t]{2}{*}{ N3 } & $250 \mathrm{~m}$ & $10^{-15}$ & \multirow{2}{*}{$10^{-13}$} & \multirow{2}{*}{0.5587} \\
\hline & & $250 \mathrm{~m}$ & $2 \cdot 10^{-13}$ & & \\
\hline
\end{tabular}

The transmission of $10 \mathrm{~Gb} / \mathrm{s}$ 4, 16 and 64-QAM signals over combined RoF and RoFSO under the six turbulence scenarios (see $\mathrm{U}$ and $\mathrm{N}$ profiles along link depicted in Fig. 2) was then simulated. The calculated EVM performances are shown in Fig. 3. As depicted in Fig. 3(a), 4-QAM modulation format leads to higher EVM values than higher-order QAM formats. Moreover, such degradation is directly related to the magnitude of averaged $C_{n}^{2}$ parameter with minor differences due to spatial distributions. In fact, there is only $1 \% \mathrm{EVM}$ deterioration between scenarios with similar averaged $C_{n}^{2}(\mathrm{U} 2, \mathrm{~N} 1, \mathrm{~N} 2$ and N3) at $0 \mathrm{dBm}$ received optical power. Note that $17.5 \%$ EVM threshold [23] for 4-QAM is fulfilled under U3 scenario with optical received power better than $0.5 \mathrm{dBm}$ whereas maximum EVM difference between scenario U1 and U3, representing low and high turbulence scenarios, respectively, at $0 \mathrm{dBm}$ of received optical power is about $6 \%$.

However, Fig. 3(b) shows a clear distinction of the 16-QAM results obtained for uniform turbulence scenario U2 and non-uniform scenarios N1, N2 and N3 with $4.6 \%$ maximum EVM deterioration between them at $0 \mathrm{dBm}$ received optical power. In this case, the uniform distribution $\mathrm{U} 2$ with moderate turbulence strength leads to higher degradation 
in comparison with similar averaged but nonuniform turbulence N1, N2 and N3 scenarios. Similarly to the results shown in Fig. 3(a), U1 and U3 are identified as the best and worst scenarios due to the weak and strong turbulence characteristics, respectively but with a larger EVM deterioration, i.e. $6.9 \%$.
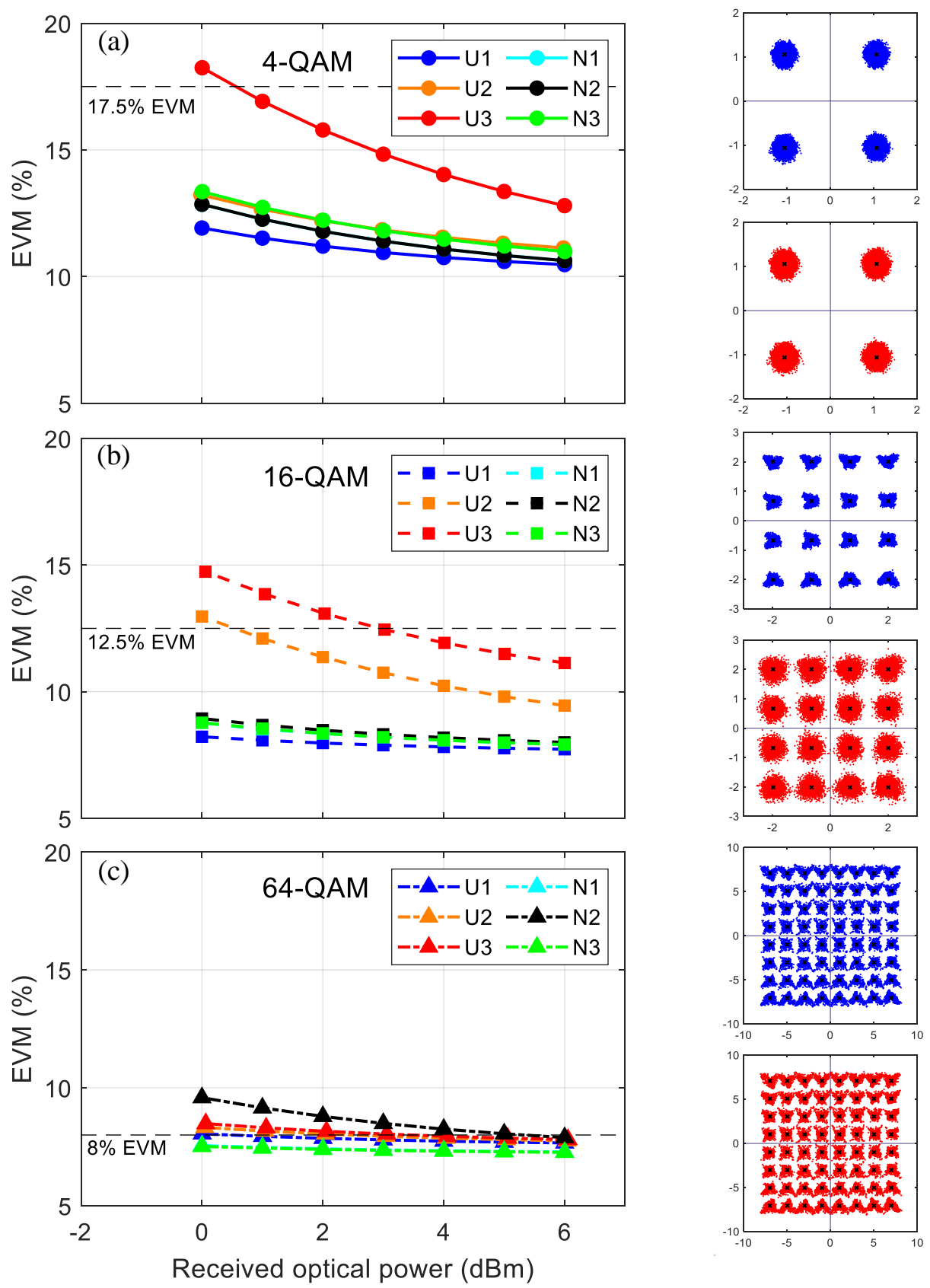

Fig. 3. EVM vs received optical power for (a) 4-QAM, (b) 16-QAM, (c) 64-QAM; insets show constellation diagrams for U1 and U3 scenarios under received optical power of $4 \mathrm{dBm}$.

Fig. 3(c) then shows EVM results when 64-QAM signal is transmitted in proposed system. We can observe that differences due to non-uniform spatial distribution of turbulence become relevant and higher 64-QAM signal sensitivity to non-uniform turbulence profile when high turbulence magnitude in the middle of the link is applied. In this case, N3 and N1, having increased turbulence levels in the first and second half of the channel, respectively, lead to better results than uniform U2, whereas N2, having a 
turbulence magnitude peak in the middle of the channel, reaches higher values than strongest uniform distribution U3. However, the maximum observed EVM penalty between the best and worst scenarios (i.e. $\mathrm{N} 3$ and $\mathrm{N} 2$, respectively) is only $2 \%$ at $0 \mathrm{dBm}$ of received optical power. Insets on the right in Fig. 3 show the constellations for low (U1) and high (U3) turbulence obtained for every modulation format when the optical received power is $4 \mathrm{dBm}$.

In order to better demonstrate the impact of variable turbulence distribution, Fig. 4 shows the EVM results for U1, U3, N2 and N3 scenarios separately. In all of them, we observe that 4-QAM shows higher EVM values, whereas 16- and 64-QAM keep EVM under $9 \%$ (U1 and $\mathrm{N} 3$ ), $10 \%$ (N2) for received optical power of $0 \mathrm{dBm}$. M-QAM evaluated formats satisfy their EVM quality threshold at $0 \mathrm{dBm}$ of received optical power. However 64-QAM transmission in N2 scenario requires a minimum received power of $4 \mathrm{dBm}$ and the signals under $\mathrm{U} 3$, which was the strongest turbulence scenario in our investigation, require corresponding threshold power values of $0.5,3$ and $3.5 \mathrm{dBm}$ for 4-, 16 and 64-QAM, respectively.
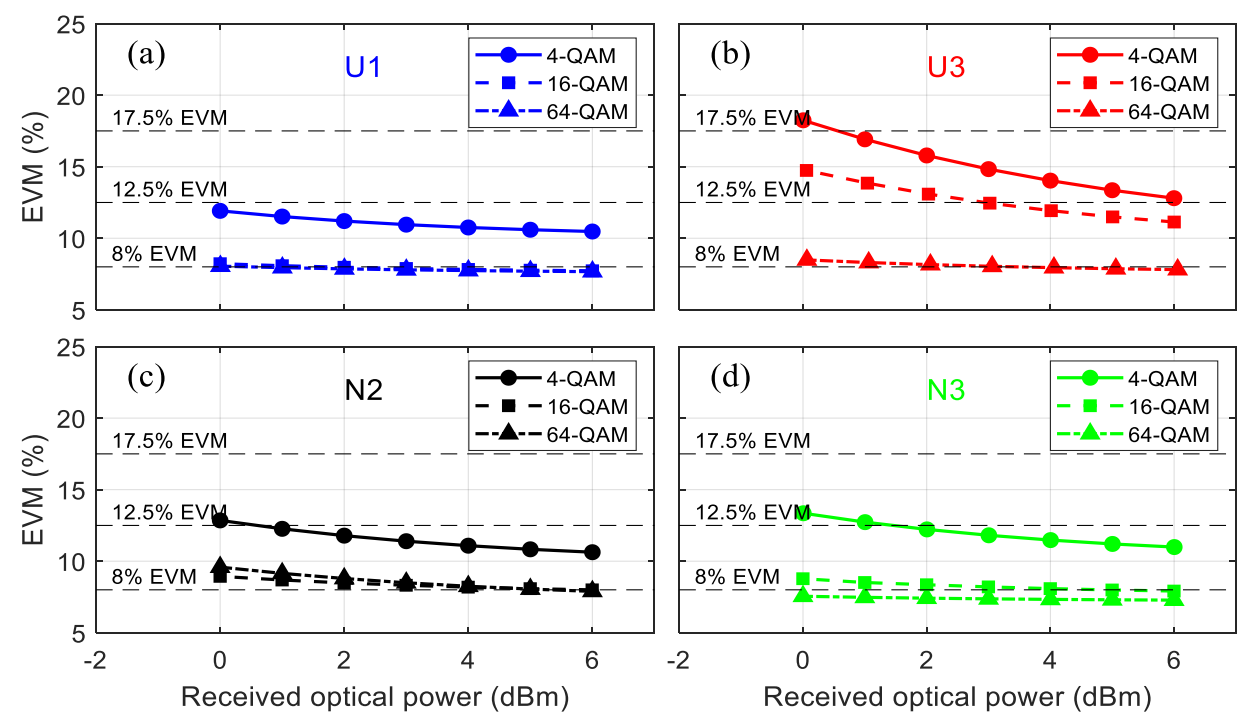

Fig. 4. Comparison of EVM vs received optical power for different scenarios: (a) U1, (b) U3, (c) N2, and (d) N3.

\section{Experimental results}

In this section, we report the experimental measurements of the impact of non-uniform turbulent distribution in RoFSO links on 4- and 64-QAM signals transmitted over $25 \mathrm{GHz}$ along a hybrid network. The experimental setup (shown in Fig. 5) follows the schematic shown in Fig. 1. A $12.5 \mathrm{GHz}$ signal generator (SG) (R\&S SMF100A) was used to drive the MZM-1 (Fujitsu FTM7938EZ/201) and a vector signal generator (VSG) (R\&S SMW200A) was employed to generate $20 \mathrm{MHz}$ M-QAM signals to drive the MZM-2 (Covega 10TM 081). An EDFA (CEFA-C-HG-SM-50-B130-FA-FA) with $13.2 \mathrm{dBm}$ constant output power was employed to compensate for optical losses. The $5 \mathrm{~km}$ of SSMF and $2 \mathrm{~m}$ FSO long links were adopted as the hybrid optical network. In order to set the FSO link, a pair of air-spaced doublet collimators (Thorlabs 114 F810APC-1550) was used what led to $4 \mathrm{~dB}$ of overall optical loss in the wireless segment. Turbulences were generated in a controlled temperature chamber by using two heating fans. Then, the optical signal was launched into a 90/10 optical coupler allowing the monitoring of the signal by the optical spectrum analyzer (OSA). Finally, an optical variable attenuator allowed adjusting the received power just before detection by a photoreceiver (OptiLab PD-40). Postamplification and further signal processing allowed to evaluate the signal quality and recover the signal constellation at $25 \mathrm{GHz}$ in the RF spectrum analyzer (RFSA) (R\&S FSW26). 


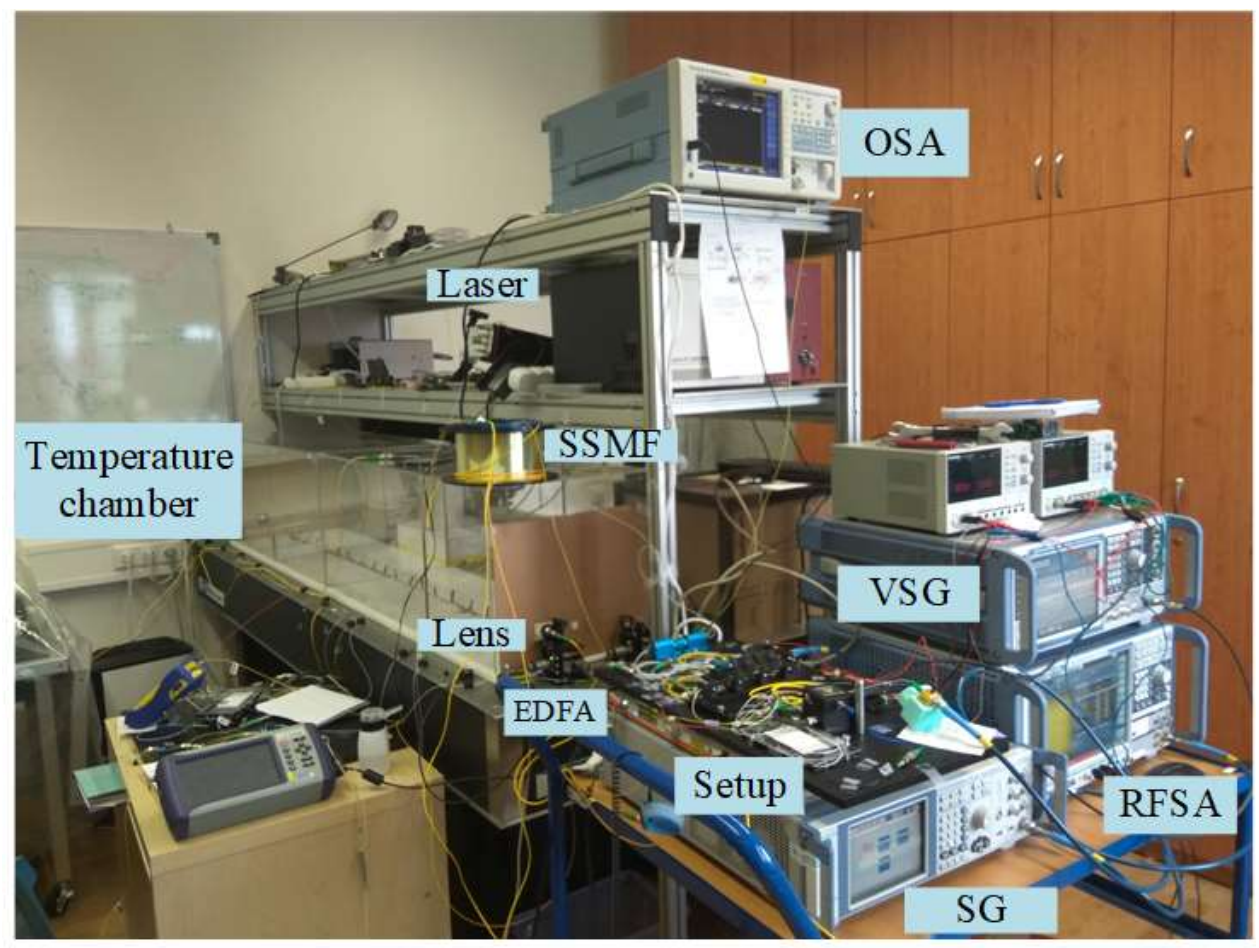

Fig. 5. Photograph of the experimental setup.

Three experimental turbulence scenarios of the FSO link D1, D2 and D3 were created in a laboratory chamber where thermal distribution was captured by thermal sensors equidistantly spaced in $10 \mathrm{~cm}$ steps and corresponding refractive index structure parameter, $C_{n}^{2}$ was calculated along the link (see distributions in Fig. 6). While almost flat distribution was set in case of D1 with $\mathrm{C}_{\mathrm{n}}^{2}=5.9 \cdot 10^{-14} \mathrm{~m}^{-2 / 3}\left(\sigma_{R}^{2}=1.3254 \cdot 10^{-5}\right.$, low turbulence), D2 and D3 correspond to turbulence distributions with a turbulence peak in the middle of the link and increased turbulence in the last part of the link, similar to N2 and $\mathrm{N} 3$, respectively, both analysed in the previous section. The averaged $C_{n}^{2}$ values were $4.2 \cdot 10^{-11} \mathrm{~m}^{-2 / 3}$ and $1.8 \cdot 10^{-11} \mathrm{~m}^{-2 / 3}$ and therefore corresponding $\sigma_{R}^{2}=0.0094754$ and $\sigma_{R}^{2}=0.0041495$ for experimental D2 and D3 scenarios, respectively. Note that lower $\sigma_{R}^{2}$ values and higher $C_{n}^{2}$ values comparing to the simulation results were obtained due to considerably shorter FSO path, i.e. $2 \mathrm{~m}$ long, in the experiment.

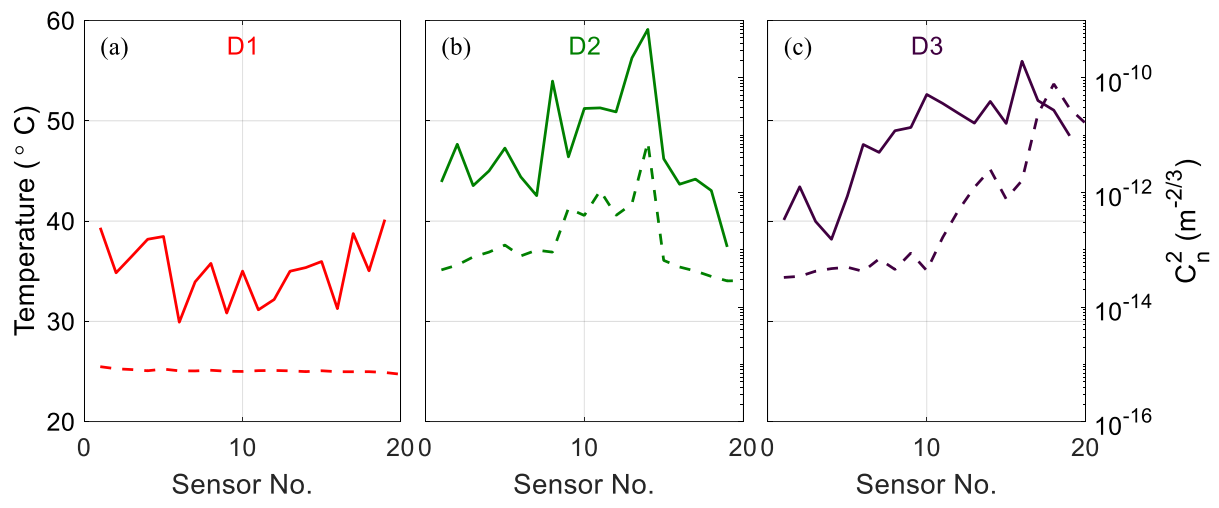

Fig. 6. Experimental temperature (dashed line) and $C_{n}^{2}$ (solid line) distributions for different scenarios: (a) D1, (b) D2 and (c) D3. 
A $25 \mathrm{GHz}$ signal was photonically generated and the signal phase noise was measured, as shown in Fig. 7 with values below $-104.8 \mathrm{dBc} / \mathrm{Hz}$ under all three turbulence scenarios at $100 \mathrm{kHz}$ frequency offset, as detailed in the inset.

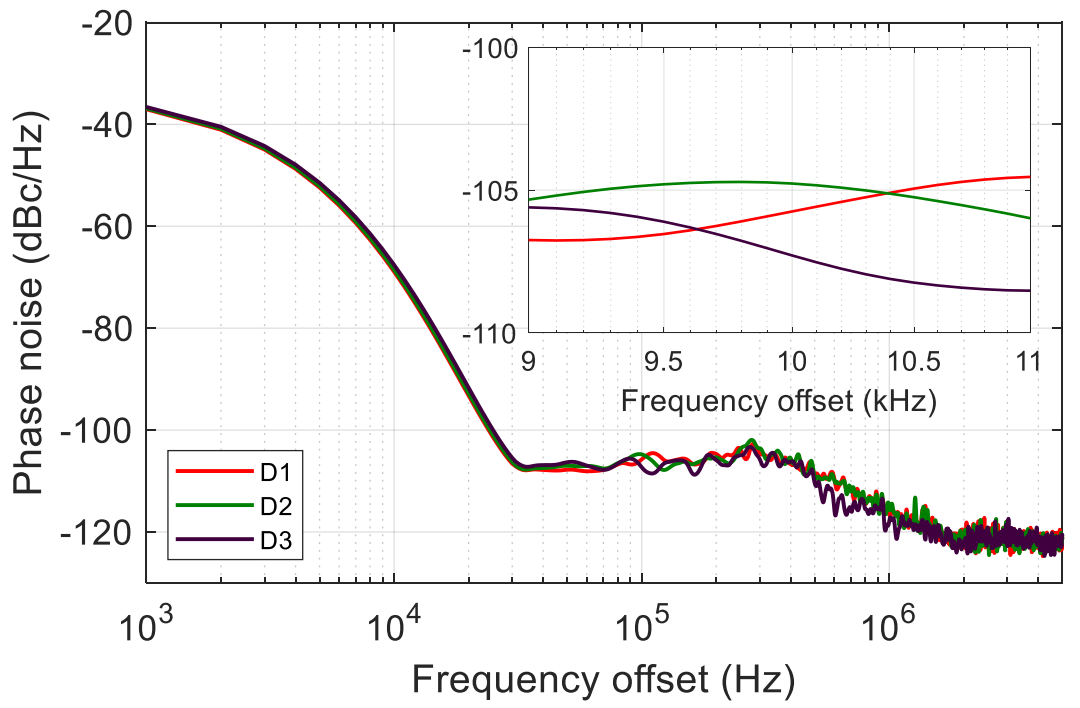

Fig. 7. Phase noise measurements for different scenarios.

In the following, 4- and 64-QAM modulated signals were transmitted along the experimental system to evaluate the impact of variable turbulence distributions on the different modulation formats. Fig. 8 shows the constellations of both recovered signals with corresponding EVM values after detection with $0 \mathrm{dBm}$ of received optical power. Although D3 scenario experienced large degradation, the data signal has been effectively analyzed in all cases.
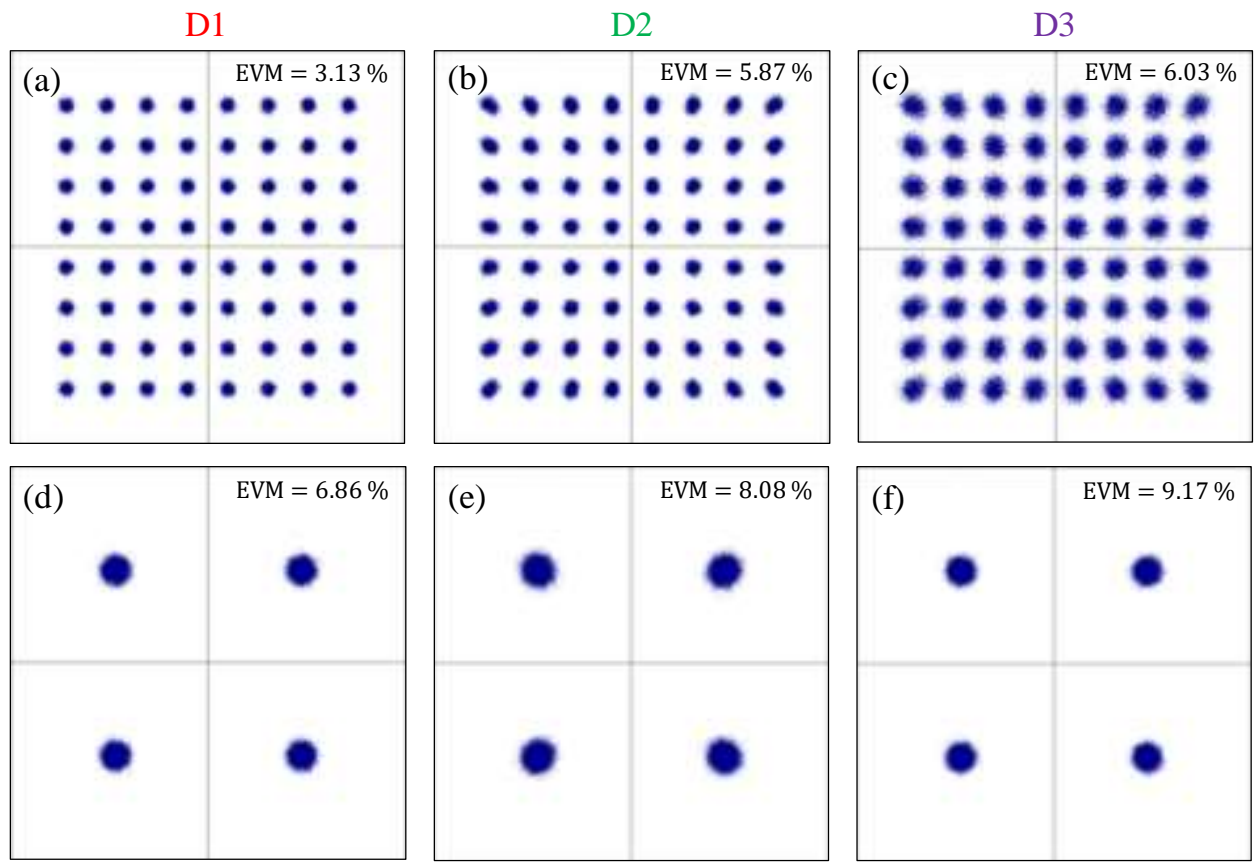

Fig. 8. Constellations at $0 \mathrm{dBm}$ received optical power: (a)-(c) 64-QAM, and (d)-(f) 4QAM for D1, D2 and D3 turbulence distributions. Corresponding EVM values are displayed in the constellations. 
Finally, Fig. 9(a) shows the measured EVM versus the received optical power for 4and 64-QAM. We can observe that EVM values are higher for 4-QAM, as obtained in the simulation results. Nevertheless the 4-QAM has significantly higher EVM limit to obtain reliable transmission. Both modulation formats lead to less degradation under low turbulence (D1 scenario) and similar degradations for D2 and D3, as expected due to the similar turbulence level magnitude. Standard EVM limits, displayed as the dashed horizontal lines, for 4- and 64-QAM are satisfied under D1 scenario with a received optical power of $-5 \mathrm{dBm}$ and $-4.33 \mathrm{dBm}$, respectively. However, the power penalties with respect to D2 and D3 scenarios at the $17.5 \% \mathrm{EVM}$ level for 4-QAM are $1.3 \mathrm{~dB}$ and $1.8 \mathrm{~dB}$, respectively, whereas the power penalties at the $8 \%$ EVM level for 64-QAM are $1.9 \mathrm{~dB}$ and $1.7 \mathrm{~dB}$. Accordingly with the simulation results shown in Fig. 3, the scenario with increased turbulence level in the last part of the link, i.e. N3, shows larger EVM than the one with the peak turbulence in the middle, i.e. N2, for 4-QAM whereas N2 exhibited larger EVM than N3 for 64-QAM. On the other hand, note that D2 and D3 scenarios with $0 \mathrm{dBm}$ received optical power lead to 3.7 and $4 \%$ EVM difference between 4- and 64-QAM formats, respectively, in good agreement with $4 \%$ penalty calculated under $\mathrm{U} 1$, with similar Rytov coefficient, in section 2.

Fig. 9 (b) shows the EVM results in terms of received electrical power measured at 25 $\mathrm{GHz}$ where 4- and 64-QAM required a minimum electrical power of -65.7 and $-66.1 \mathrm{dBm}$, respectively showing penalties lower than 0.94 and $1.7 \mathrm{~dB}$ for D2 and D3 in both cases.
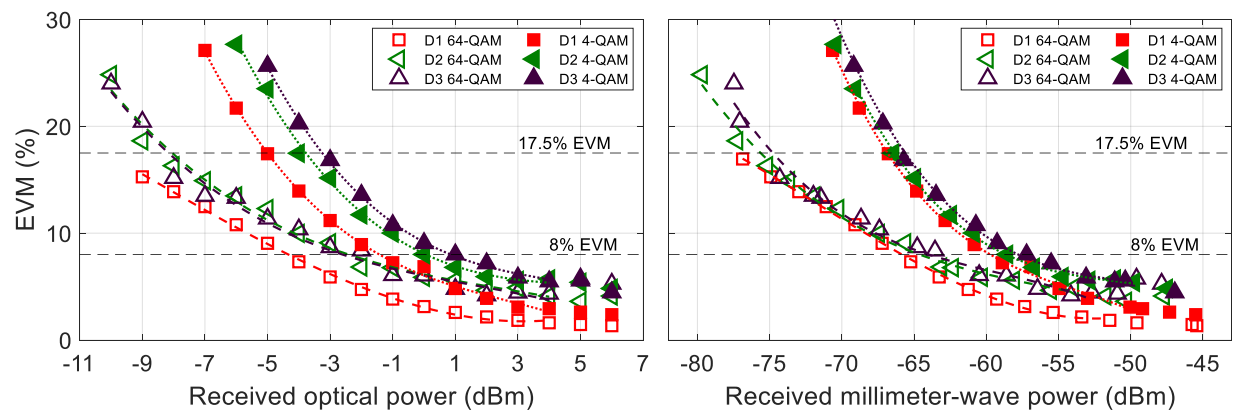

Fig. 9. Experimental EVM vs received optical (a) and electrical (b) power for 4-QAM and 64-QAM modulation formats.

Fig. 10 shows the equivalent bit error rate (BER) levels in log scale calculated as described in [24], also showing the corresponding BER threshold levels at $5.5 \cdot 10^{-9}$ and $1.9 \cdot 10^{-3}$ for 4 - and 64-QAM, respectively. Note that BER level decrease with increasing received power changes such behavior at high power values, e.g. $3-7 \mathrm{dBm}$ due to nonlinear detector response.
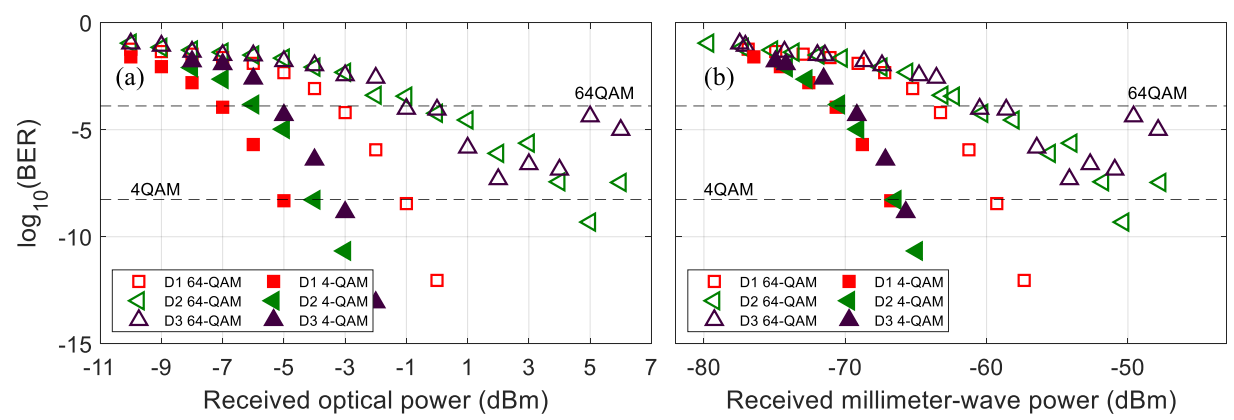

Fig. 10. Experimental BER vs received optical (a) and electrical (b) power for 4-QAM and 64-QAM modulation formats.

\section{Conclusions}

We have theoretically and experimentally evaluated the impact of different thermalinduced free space turbulence distributions on the M-QAM signal transmission in photonically generated K-band carrier over hybrid optical links. Simulation results of 
$10 \mathrm{~Gb} / \mathrm{s}$ signal with 4-, 16- and 64-QAM over $5 \mathrm{~km}$ of SSMF and $500 \mathrm{~m}$ long FSO link have been demonstrated under different weak to strong turbulence regimes. Non-uniform turbulence distributions are shown to have different impacts on the transmission of $\mathrm{M}$ QAM modulation formats in given frequency band with practical consequences. Moreover, real transmission experiments of $20 \mathrm{MHz} 4$ - and 64- QAM signals over $5 \mathrm{~km}$ of SSMF and $2 \mathrm{~m}$ long FSO link confirm that turbulence distributions with higher strength in the middle of the considered link have bigger impact in terms of power penalty on 64-QAM (1.9 dB penalty) compared to 4-QAM (1.3 dB penalty). For 4-QAM, higher EVM results have been measured for distributions with larger turbulence magnitude in the second half of the link what is in good agreement with simulation results. In spite of different theoretical and experimental bitrates and FSO link length, results agree on $4 \%$ EVM difference between 4- and 64-QAM at a given received optical power, i.e. $0 \mathrm{dBm}$, when compared equivalent links with uniform weak turbulence. On the other hand, the presented system needs careful attention when the FSO link is exposed to optical turbulences with high gradients along the optical path, e.g. in dense urban areas. Further work can be done towards the experimental signal transmission over non-uniform scenarios under moderate and strong regimes to evaluate larger EVM differences.

\section{Funding}

This work was also supported by the Research Excellence Award Programme GVA PROMETEO 2017/103, the Spanish Ministerio de Ciencia, Innovación y Universidades RTI2018-101658-B-I00 FOCAL project, Ministry of Industry and Trade in Czech Republic project (FV30427) and within COST CA16220.

\section{References}

1. R. Zhang, F. Lu, M. Xu, S. Liu, P.-C. Peng, S. Shen, J. He, H. J. Cho, Q. Zhou, S. Yao and G.-K. Chang, "An ultra-reliable MMW/FSO A-Rof system based on coordinated mapping and combining technique for 5G and beyond mobile fronthaul," J. Lightw. Technol. 36(20), 4952-4959 (2018)

2. ETSI, "TS 38.101-2 V 15.5.0. 5G; NR; User Equipment (UE) radio transmission and reception; Part 2: Range 2 Standalone," (2019).

3. C. H. Lee, Microwave Photonics (CRC Press, 2013), 2nd ed.

4. A. Checko, H. L. Christiansen, Y. Yan, L. Scolari, G. Kardaras, M. S. Berger and L. Dittmann, "Cloud RAN for mobile networks - A technology overview," IEEE Commun. Surveys Tuts. 17(1), 405-426 (2015).

5. C. Lim, Y. Tian, C. Ranaweera, T. A. Nirmalathas, E. Wong and K.-L. Lee, "Evolution of Radio-OverFiber Technology," J. Lightw. Technol. 37(6), 1647-1656 (2019).

6. Y. Doi, S. Fukushima, T. Ohno and K. Yoshino, "Frequency stabilization of millimeter-wave subcarrier using laser heterodyne source and optical delay line," IEEE Photon. Technol. Lett. 13(9), 1002-1004 (2001).

7. J. Yao, "Microwave photonics," J. Lightw. Technol. 27(3), 314-335 (2009).

8. H. Zhang, L. Cai, S. Xie, K. Zhang, X. Wu and Z. Dong, "A novel radio-over-fiber system based on carrier suppressed frequency eightfold millimeter wave generation," IEEE Photon. J. 9(5) (2017)

9. M. A. Khalighi and M. Uysal, "Survey on free space optical communication: a communication theory perspective," IEEE Commun. Surveys Tuts. 16(4), 2231-2258 (2014).

10. S. Bloom, E. Korevaar, J. Schuster and H. Willebrand, "Understanding the performance of free-space optics [Invited]," J. Opt. Netw. 2(6), 178-200 (2003).

11. H. R. Anderson, Fixed Broadband Wireless System Design (Wiley, 2003), 1 st ed.

12. Z. Ghassemlooy, W. Popoola and S. Rajbhandari, Optical wireless communications. System and channel modelling with Matlab (CRC Press Taylor \& Francis group, 2019), 2nd ed.

13. D. K. Borah and D. G. Voelz, "Pointing Error Effects on free-space optical communication links in the presence of atmospheric turbulence," J. Lightw. Technol. 27(18), 3965-3973 (2009).

14. M. A. Esmail, A. Ragheb, H. Fathallah and M.-S. Alouini, "Investigation and demonstration of high speed full-optical hybrid FSO/fiber communication system under light sand storm condition," IEEE Photon. J. 9(1) (2017).

15. J. Libich and S. Zvanovec, "Influences of turbulences in near vicinity of buildings on free-space optical links," IET Microw., Antennas P. 5(9), 1039-1044 (2011).

16. K. Niachou, I. Livada and M. Santamouris, "Experimental study of temperature and airflow distribution inside an urban street canyon during hot summer weather conditions. Part II: Airflow analysis," Build. Environ. 43(8), 1393-1403 (2008)

17. D.-N. Nguyen, J. Bohata, J. Spacil, D. Dousek, M. Komanec, S. Zvanovec, Z. Ghassemlooy and B. Ortega, "M-QAM transmission over hybrid microwave photonic links at the K-band," Opt. Express 27(23), 33745-33756 (2019). 
18. D.-N. Nguyen, J. Bohata, M. Komanec, S. Zvanovec, B. Ortega and Z. Ghassemlooy, "Seamless 25 GHz transmission of LTE 4/16/64-QAM signals over hybrid SMF/FSO and wireless link," J. Lightw. Technol. 37(24), 6040-6047 (2019).

19. L. Vallejo, M. Komanec, B. Ortega, J. Bohata, D.-N. Nguyen, S. Zvanovec and V. Almenar, "Impact of thermal-induced turbulent distribution along FSO link on transmission of photonically generated $\mathrm{mmW}$ signals in the frequency range 26-40 GHz," IEEE Photon. J. 12(1) (2020).

20. G. Qi, J. Yao, J. Seregelyi, S. Paquet, C. Belisle, X. Zhang, K. Wu and R. Kashyap, "Phase-noise analysis of optically generated millimeter-wave signals with external optical modulation techniques," J. Lightw. Technol. 24(12), 4861-4875 (2006).

21. J. Ma, J. Yu, C. Yu, X. Xin, J. Zeng and L. Chen, "Fiber dispersion influence on transmission of the optical millimeter-waves generated using LN-MZM intensity modulation," J. Lightw. Technol. 25(11), 3244-3256 (2006).

22. L. C. Andrews and R. L. Phillips, Laser Beam Propagation through Random Media (SPIE Press, 2005) 2nd ed.

23. ETSI, “TS 138.101-1 V15.5.0. User Equipment (UE) radio transmission and reception; Part 2: Range 2 Standalone," (2019).

24. X. Chen and J. Yao, "A high spectral efficiency coherent microwavephotonic link employing both amplitude and phasemodulation with digital phase noise cancellation," J. Lightw. Technol. 33(14), 30913097 (2015). 